\title{
Determinación de la biomasa de las poblaciones bacterianas fotosintéticas en la laguna de Ciso (Banyoles, Girona)
}

\author{
Jordi Mas
}

Departamento de Microbiología, Facultad de Ciencias

Universidad Autónoma de Barcelona.

\author{
SUMMARY \\ BIOMASS DETERMINATION OF PHOTOSINTHETIC BACTERIAL POPULATIONS \\ IN CISÓ POND (BANYOLES, NE SPAIN)
}

Two biochemical methods (ATP, pigments) and one microscopical method (Acridine Orange Direct Count Technique) have been utilized for estimating bacterial biomass in vertical profiles of an holomictic lake (Cisó) located in the karstic system of Banyoles (Girona, NE Spain). The highest biomass values have been obtained with the pigment procedure and the lowest with the ATP method. Differences are discussed and a new C/ATP ratio of 375 is proposed, in this case, in order to prevent underestimation made by the ATP method.

\section{INTRODUCCION}

El estudio de las poblaciones bacterianas en la naturaleza adquiere especial relieve desde el momento en que se pone en evidencia su elevada relación actividad/biomasa y como consecuencia, su incidencia en los procesos biogeoquímicos de reciclado de los elementos.

Las peculiares características físico-quí: micas de la laguna de Cisó hacen que predominen poblaciones de bacterias fotosintéticas correspondientes fundamentalmente a los géneros Chromatium y Chlorobium (GUERRERO \& ABELLA, 1978). Estas bacterias, que se encuentran en el agua en cantidades elevadas, son las responsables de prácticamen- te la totalidad de la actividad fijadora de carbono del sistema (MONTESINOS, 1982). Este hecho hace que la determinación precisa de la biomasa sea necesaria para definir de manera correcta el esquema global de funcionamiento de la laguna estudiada.

La determinación de la biomasa bacteriana en la naturaleza constituye un problema importante que se ha intentado resolver por dos caminos distintos. Por una parte se han desarrollado una serie de procedimientos directos basados en recuentos microscópicos por diferentes métodos: microscopía óptica convencional, microscopía óptica de epifluorescencia (HOBBIE et al., 1977) y microscopía electrónica (PAERL \& SHIMP, 1973). Paralelamente, ha aparecido un conjunto de 
técnicas indirectas de tipo bioquímico que estiman la biomasa cuantificando algún componente celular con el que ésta se encuentre estrechamente relacionada. A este segundo grupo pertenecen los métodos basados en la detección y cuantificación de mucopolisacáridos (MORIARTY; 1975), lipopolisacáridos (WATSON, et al. 1977), de ATP (HOLMHANSEN \& BOOTH, 1966) de proteínas (COHEN et al., 1977) y, en el caso de orgaticos, de pigmentos (TRÜPER, 1967). nismos fotosintéticos, de pigmentos (TRÜPER \& YENTSCH 1967).

El estudio que hemos llevado a cabo ha consistido en la determinación periódica de la biomasa bacteriana siguiendo tres de los procedimientos antes citados: cuantificación de pigmentos, ATP y número total de células calculado por microscopía de epifluorescencia.

\section{MATERIAL Y METODOS}

\section{LUGAR DE ESTUDIO}

El lugar donde hemos llevado a cabo este estudio ha sido la laguna de Cisó, situada en el sistema cárstico de Banyoles, en la provincia de Girona. Se trata de una laguna anaerobia de aguas sulfurosas y dimensiones reducidas, cuyas características han sido descritas en otros trabajos (GUERRERO \& ABELLA, 1978; GUERRERO et al., 1980).

\section{MUESTREO Y CONSERVACION DE LAS MUESTRAS}

Las muestras fueron obtenidas a distintas profundidades utilizando una botella limnológica de Ruttner de 1,9 litros de capacidad, inmediatamente fueron transportadas al laboratorio en condiciones de oscuridad y a $4^{\circ} \mathrm{C}$ para su posterior análisis.

\section{RECUENTO DIRECTO}

Para la fijación de las muestras se utilizó formaldehido a una concentración final del $0,02 \%$. A continuación fueron filtradas y teñidas sobre filtros Nuclepore de $0,2 \mathrm{mi}$ cras de diámetros de poro según la técnica de Hobbie (HOBBIE et al., 1977). Para las observaciones se utilizó un microscopio Olympus modelo $\mathrm{BH}$. La estimación de la biomasa a partir de los números totales observados se hizo teniendo en cuenta los volúmenes celulares medios calculados geométricamente a partir de imágenes de microscopia electrónica (tabla I). Se consideró como densidad celular media $1,07 \mathrm{~g} / \mathrm{cm}^{3}$ y el peso fresco obtenido (pf) se transformó a gramos de carbono mediante la relación $\mathrm{pf} / \mathrm{C}=8,5$.

\section{EXTRACCION Y MEDIDA DE ATP}

Las muestras fueron concentradas en filtros de membrana de 0,45 micras de diáme tro medio. La extracción se efectuó por ebullición del filtro en tampón Tris-HC1 $0,025 \mathrm{M}$ a $\mathrm{pH} 7,75$ durante 5 minutos. El extracto se conservó a $-20^{\circ} \mathrm{C}$ hasta el momento del ensayo.

Para el ensayo se utilizó extracto enzimático liofilizado de luciferina-luciferasa de luciérnaga (Sigma) rehidratada en el mismo tampón utilizado para la extracción e incubada en la oscuridad y a temperatura ambiente durante 3-4 horas antes de su utilización. La emisión de luz se midió por medio de un aparato Berthold Biolumat LB 9.500 acoplado a una calculadora impresora HP-97 de Hewlett-Packard para el procesado de los datos. En cada una de las muestras se utilizó un estándar interno de ATP (marca LKB) de concentración conocida a fin de controlar los efectos de inhibición de la reacción debidos a "quenching" o a alteraciones de la actividad enzimática. La conversión de ATP a biomasa en carbono se realizó utilizando la relación propuesta por Holm-Hansen C/ATP $=250$ (HOLM-HANSEN \& BOOTH 1966).

\section{EXTRACCION Y CUANTIFICACION DE PIGMENTOS}

La extracción de pigmentos se llevó a ca- 
TABLA I. Volúmenes celulares medios y frecuencias relativas en diferentes épocas del año, de las principales especies de bacterias fotosintéticas observadas en la laguna de Cisó. +Porcentaje referido al total de la población presente en la fecha indicada.

Especie

Chromatiaceae

Chromatium minus

Thiocystis violacescens

Chlorobiaceae

Chtorobium limicola

chlorobium phaeobacteroides

chlorobizm vibrioforme

Pelodictyon clatharatiforme

bo filtrando las muestras sobre filtros de membrana de $0,45 \mu \mathrm{m}$ de diámetro de poro recubiertos de una capa de carbonato magnésico. El carbonato magnésico con las células fue raspado $y$ resuspendido en acetona a $4^{\circ} \mathrm{C}$ durante una noche para la total extracción de los pigmentos, con centrifugación posterior a $7.000 \mathrm{~g}$. durante 10 minutos.

La absorbancia del sobrenadante se midió con un espectrofotómetro Pye Unicam SP 1.700 a 654 y $773 \mathrm{~nm}$, que son los máximos de absorción en acetona de las bacterioclorofilas $c$ y $a$ respectivamente. Los valores obtenidos se convirtieron a concentración de pigmentos utilizando las fórmulas de Takahashi y Ichimura (TAKAHASHI \& ICHIMURA, 1970) y posteriormente a biomasa en carbono utilizando las ecuaciones calculadas empíricamente durante el período 1978-1979 para las dos bacterioclorofilas presentes (MONTESINOS, 1982).

\section{RESULTADOS Y DISCUSION}

La mayor parte de los muestreos corresponden al período de holomixis es decir con heterogeneidad vertical muy atenuada, por lo que eran de esperar valores homogéneos para toda la columna de agua. Los resultados de biomasas en $\mu \mathrm{gC} / 1$ para cada día $\mathrm{y}$ profundidad, calculados por las técnicas de ATP, recuento total y pigmentos se indican
Volumen (um )

\author{
$21-8-81$
}

$(\%)^{+}$

$23-12-81$

$(\%)+$

8,39
1,48
1,03
0,53
0,25
0,29

78,0

14,6

1,48

8,8

51,7

1,03

0,29

$\begin{array}{cc}4,2 & 33,1 \\ - & 0,6 \\ - & -\end{array}$

en la tabla II. En la figura 1 se puede observar la variación, en función del tiempo, de la biomasa integrada del perfil vertical según los distintos procedimientos.

Los valores más elevados son los calculados a partir de la concentración de pigmentos; les siguen los calculados por recuento directo; $\mathrm{y}$, por último, se encuentran los obtenidos por la técnica del ATP. Esta diferencia se mantiene a lo largo de todo el período de estudio. Para ver si las discrepancias entre los métodos eran estadísticamente significativas, se contrastaron las medias muestrales utilizando el test de " $t$ " para observaciones apareadas (tabla III). Los resultados de esta prueba reafirman las observaciones preliminares, confirmando las

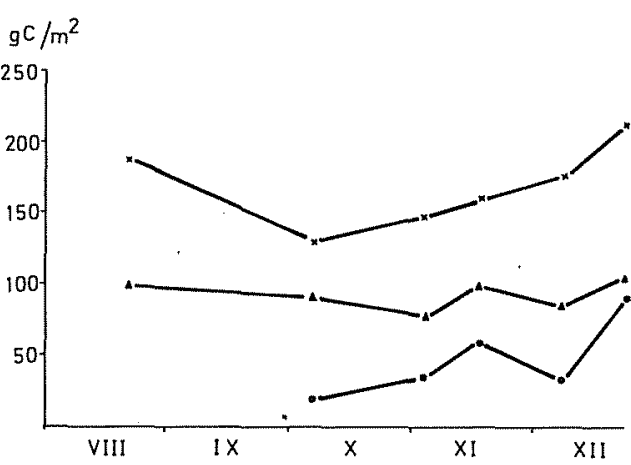

Fig. 1. Variación según el tiempo de los perfiles verticales integrados de biomasa durante el periodo de estudio, (agosto-diciembre de 1981), utilizando los tres métodos. X Pigmentos; $\Delta$ Recuento microscópico; ATP. 
TABLA II. Valores de biomasa ( $\mu \mathrm{g}$ C/1) para los diferentes dias, profundidades y técnicas empleadas. (a): recuento directo por microscopía de epifluorescencia. (b): calculada a partir de pigmentos. (c): calculada a partir de ATP.

\begin{tabular}{|c|c|c|c|c|c|c|c|c|c|c|c|c|c|c|}
\hline \multirow{2}{*}{$\frac{\text { Prof. }}{(\mathrm{m})}$} & \multicolumn{2}{|c|}{$21-8-81$} & \multicolumn{3}{|c|}{$6-10-81$} & \multicolumn{3}{|c|}{$3-11-81$} & \multicolumn{3}{|c|}{$7-12-81$} & \multicolumn{3}{|c|}{$23-12-81$} \\
\hline & Micr. ${ }^{a}$ & Pigm. ${ }^{b}$ & Micr. & Pigm. & $\operatorname{ATP}^{C}$ & Micr. & Pigm. & $\mathrm{ATP}$ & Micr. & Pigm. & $\Lambda T P$ & Micr. & Pigm. & $\triangle T P$ \\
\hline 0 & 541 & 939 & -- & -- & -- & -- & -- & -- & 1002 & 1670 & 382 & -- & -- & -- \\
\hline 0,25 & -- & -- & 873 & 1624 & 47 & 960 & 1.338 & 198 & -- & -- & -- & 848 & 1941 & 698 \\
\hline 0,50 & 2404 & 3666 & 747 & 2112 & 532 & -- & -- & $\ldots .$. & 879 & 1739 & 4.13 & 994 & 1909 & 609 \\
\hline 0,75 & -- & -- & -- & -- & -- & 618 & 1385 & 113 & 845 & 1666 & 425 & -- & -- & -- \\
\hline 1 & 1982 & 2729 & -- & -- & -- & -- & -- & -- & 692 & 1757 & 222 & -- & -- & -- \\
\hline 1,50 & 843 & 1647 & 743 & 1429 & 58 & 409 & 1353 & 289 & -- & -- & -- & 1005 & 1941 & 748 \\
\hline 3 & 673 & 1362 & -- & -- & -- & -- & -- & -- & -- & -- & -- & 882 & 191.5 & 859 \\
\hline 4 & -- & -- & -- & -- & - & -- & -- & -- & 961 & 1726 & 268 & -- & - & -- \\
\hline 5 & -- & -- & 895 & 915 & 92 & 600 & 1308 & 169 & -- & -- & - & -- & -- & -- \\
\hline 8 & 871 & 1347 & -- & -- & -- & -- & -- & -- & -- & -- & -- & 754 & 1977 & 1249 \\
\hline 9 & -- & -- & -- & -- & -- & 718 & 1349 & 289 & 810 & 1654 & 310 & -- & -- & -- \\
\hline
\end{tabular}


TABLA III. Resultados del test de ' $t$ ' para observaciones apareadas, correspondientes a los datos originales. Nivel de significación: $+, 0,025 ;++, 0,001$

$\begin{array}{lcc} & \text { Pigmentos } & \text { ATP } \\ \text { No total } & 18,33++ & 2,50+ \\ \text { Pigmentos } & - & 15,67++\end{array}$

A pesar de la corrección por 0,5 y del mejor ajuste que esta corrección implica, el diferencias entre los resultados obtenidos por los tres procedimientos.

Teniendo en cuenta las desviaciones antes comentadas, se aplicaron unos factores de corrección arbitrarios de 0,5 para la biomasa calculada mediante pigmentos y 1,5 para la derivada del ATP. Los datos corregidos de este modo fueron sometidos de nuevo al test de " $t$ " para observaciones apareadas (tabla IV), con lo cual no se apreciaron diferencias significativas entre los métodos.

La sobreestimación de la biomasa calculada a partir de la concentración de pigmentos se debe a la aparición durante el periodo de estudio de una especie (probablemente Thiccystis gelatinosa) no descrita con anterioridad en la laguna de Cisó. Esta bacteria posee un contenido especifico de pigmentos mayor que el de las especies predominantes en el periodo 1978-1979, durante el cual se elaboraron las ecuaciones de conversión utilizadas en nuestro trabajo.

En lo que se refiere a los datos de biomasa estimados a partir del ATP, es conveniente tener en cuenta que el factor de conversión utilizado $\mathrm{C} / \mathrm{ATP}=250$ es el calculado por Holm-Hansen a partir de 7 cepas de bacterias marinas y 30 especies de algas unicelulares (HOLM-HANSEN \& BOOTH, 1966). En el caso de un sistema hipertrófico como el que estamos estudiando, el factor de conversión real puede no coincidir con el teórico utilizado.
TABLA IV. Resultados del test de 't' para observaciones apareadas, correspondientes a los datos cocorregidos. $n s=$ no significativo.

$\begin{array}{lcc} & \begin{array}{c}\text { Pigmentos } \\ (x 0,5)\end{array} & \begin{array}{c}\text { ATP } \\ (x 1,5)\end{array} \\ & 0,01^{n s} & 0,29^{\text {ns }} \\ \text { № total } & - & 0,32^{\text {ns }}\end{array}$

método de cuantificación de biomasas mediante pigmentos puede inducirnos a errores debidos a variaciones frecuentes en el contenido específico de pigmentos de las especies dominantes (ESTEVE et al., 1980). En la técnica del ATP este tipo de error es mucho menos importante debido a que las variaciones en el contenido específico de ATP son menos frecuentes $y$, en cualquier caso, de menor importancia. De todos modos, su ajuste ha requerido una corrección por 1,5. Esto nos lleva a proponer como estimador de la biomasa por este método un nuevo factor de conversión $\mathrm{C} / \mathrm{ATP}=375$ $(250 \times 1,5)$, el cual se ajusta con más exactitud a las caracteristicas de las poblaciones estudiadas.

Si bien el recuento directo por microscopia de epifluorescencia es, de los tres estudiados, el procedimiento que se ve menos afectado por la composición y estado fisiológico de las poblaciones que integran el sistema, presenta el problema de que es también el más tedioso, subjetivo y propenso a errores metodológicos. Las técnicas bioquí. micas examinadas en este trabajo son más sensibles y presentan un margen de error metodológico menor, pero, al mismo tiempo, se hallan más expuestas a errores en la estimación debido a las variaciones poco previsibles de las condiciones y composición del medio. Es por esto por lo que creemos que ambos tipos de técnicas se complementan y que es conveniente aplicarlas simultáneamente a fin de aumentar la certeza de las determinăciones. 
COHEN, Y.; KRUMBEIN, W.; \& SHILO, M. 1977 Solar Lake (Sinai). 3. Bacterial distribution and production. Limnol. Oceanogr., 22: 621-634.

ESTEVE, I.; MONTESINOS, E; ABELLA, C.; \& GUERRERO, R. 1980. Changes in the ultraestructure of the purple sulfur bacteria Chromatium minus depending on the variation of light in a natural habitat. Electron Microscopy, 2: 456-457.

GUERRERO, R.; \& ABELLA, C. 1978. Dinámica espaciotemporal de las poblaciones bacterianas fotosintéticas en una laguna anaerobia de aguas sulfurosas. Oecologia aquatica. 3: 193205.

GUERRERO, R.; MONTESINOS, E; ESTEVE, I. \& ABELLA, C. 1980. Physiological adaptation and growth of purple and green sulfur bacteria in a meromictic lake (Vila) as compared to a holomictic laké (Cisó). In: Developments in Hydrobiology, 3: 161-171) (M. DOKULIL, H. METZ and D. JEWSON eds.). Dr. W. Junk Publishers, The Hague.

HOBBIE, J.E.; DALEY, R.J. \& JASPER, S. 1977. Use of Nuclepore filters for counting bacteria by fluorescence microscopy. Appl. Environ. Microbiol., 33: 1.225-1.228.

HOLM-HANSEN, O.; \& BOOTH, C.R. 1966. The measurement of adenosine triphosphate in the ocean and its ecological significance. Limnol. Oceanogr., 11: 510-519.

MONTESINOS, E. 1982. Ecofisiologia de la fotosintesis bacteriana. Tesis Doctoral. Universidad Autónoma de Barcelona.

MORIARTY, D.J.W. 1975. A method for estimating the biomass of bacteria in aquatic sediments and its application to trophic studies. Oecologia, 20: 219-229.

PAERL, H.W., \& SHIMP, S.L. 1973. Preparation of filtered plankton and detritus for study with scanning electron microscopy. Limnol. Oceanogr., 18: 802-805.

TAKAHASHI, M. \& ICHIMURA, S. 1970, Photosynthetic properties and growth of photosynthetic sulfur bacteria in lakes. Limnol. Oceanogr., 15: 929-944.

TRUPER, H.G. \& YENTSCH, C.S. 1967. Use of glass fibre filters for the rapid preparation of in vivo absortion spectra of photosynthetic bacteria. J. Bacteriol., 94: 1.255-1.256.

WATSON, S.W.; NOVITSKY, T.J.; QUINBY, H.L. \& VALOIS, F.W. 1977. Determination of bacterial number and biomass in the marine environment. Appl. Environ. Microbiol, 33: 940946. 